\title{
Characterisation of dispersions within annealed HVOLF thermally sprayed AISnCu coatings
}

\author{
Chang-Jing Kong, Grigore Moldovan, Mike W. Fay, D. Graham \\ McCartney and Paul. D. Brown \\ School of Mechanical, Materials, Manufacturing Engineering \& \\ Management, University of Nottingham, University Park, Nottingham, \\ NG7 2RD, UK
}

\begin{abstract}
High velocity oxy-liquid fuel (HVOLF) AlSnCu coatings are characterised following annealing for up to 5 hours at $300^{\circ} \mathrm{C}$. A combination of statistical analysis of BSE images and TEM observations demonstrate the decrease in the number of sub-micron and nanoscale $\mathrm{Sn}$ particles with annealing, commensurate with a decrease in the coating microhardness. TEM evidence further suggests the coarsening of nanoscale $\mathrm{Sn}$ through a mechanism of a liquid phase migration within the Al matrix. EELS and EFTEM additionally allow the identification of the precipitation of $\theta^{\prime}$.
\end{abstract}

\section{Introduction}

The Al-12wt.\%Sn-1wt.\%Cu (A112Sn1Cu) alloy deposited by the high velocity oxyliquid fuel (HVOLF) thermally spray technique may be used for the manufacture of automotive bearings. The soft $\mathrm{Sn}$ phase acts as a self-lubricant that introduces good anti-friction characteristics. However, the microhardness of the as-sprayed coating is too high for the bearing material applications and heat treatments are applied to reduce the coating microhardness. In this paper, the microstructure of $\mathrm{Al} 12 \mathrm{Sn} 1 \mathrm{Cu}$ as-sprayed and annealed coatings is characterised, with particular regard to the dispersion and evolution of $\mathrm{Sn}$ particles and the development of $\mathrm{Al}-\mathrm{Cu}$ compound precipitates. The factors leading to the decrease in microhardness with increasing annealing time are discussed.

\section{Experimental}

The HVOLF thermal spraying parameters for the $\mathrm{Al} 12 \mathrm{Sn} 1 \mathrm{Cu}$ coating have been described previously (Kong et al 2001). The coatings were heat treated at $300^{\circ} \mathrm{C}$ for between 15 minute and 5 hours. Metallographically sectioned samples were examined using backscattered electron (BSE) imaging in a JEOL 6400 SEM. Three BSE images representing each processing condition were selected for analysis. A grey-level based threshold was applied to each and the resulting binary maps were used to measure the projected areas of the sub-micron Sn particles. A criterion of a minimum of 9 pixels was used to determine the smallest quantifiable area. Histograms of particle number against particle area, averaged across each set of images, were constructed. A Jeol 2000fx TEM was used to examine the development of the nanoscale Sn dispersion within the annealed coatings, whilst a Jeol 4000fx was used to characterise the fine details of the precipitation, using the EFTEM and EELS techniques. 



Figure 1. BSE images of HVOLF thermal sprayed coatings and statistical analysis of the sub-micron Sn particles. (a) As-sprayed coating. (b) Coating heat treated at $300^{\circ} \mathrm{C}$ for 1 hour. (c) Coating heat treated at $300^{\circ} \mathrm{C}$ for 5 hours. (d) The relationship between number of Sn particles against area as a function of time of annealing.

\section{Results and discussion}

Figures 1a-c show BSE images of Al12Sn1Cu coatings for the as-sprayed condition and annealed at $300^{\circ} \mathrm{C}$ for 1 hour and 5 hours, respectively. The light regions within these images correspond to large Sn particles, the dark regions correspond to the Al rich matrix, whilst the intermediate grey contrast regions are attributed to dispersions of nanoscale Sn within the coatings (Kong et al 2001). It is apparent that the area fraction of the grey contrast regions within these BSE images decreases as the annealing time increases. However, changes in the light contrast regions due to the effects of annealing is less easy to distinguish. Accordingly, statistical analysis of these images was performed using the ImageJ software package to produce histograms of the number of large Sn particles against project area.

Inspection of Figure 1d representing the Sn particle distributions within the assprayed coating and those annealed at $300^{\circ} \mathrm{C}$ for 1 hour and 5 hours initially shows that the Sn particle number decreases with increasing particle area, tending to zero for an area value of greater than $1.0 \mu \mathrm{m}^{2}$. The histogram shows that the number of sub-micron Sn particles with area between $0.1 \sim 0.3 \mu \mathrm{m}^{2}$ within the same volume decrease slightly after 1 hour annealing at $300^{\circ} \mathrm{C}$, as compared with the as-sprayed coating, but decreases significantly after 5 hours of annealing. For $\mathrm{Sn}$ particles of area between $0.3 \sim 0.55 \mu \mathrm{m}^{2}$, there is little difference in the decrease of the number of Sn particles within these annealed coatings. For the Sn particles with area greater than $0.55 \mu \mathrm{m}^{2}$, the number of Sn particles slightly increases after 5 hours of annealing. These results indicate that the sub-micron Sn particles tend to coalescence and merge with the larger Sn particles as the annealing time is increased. 

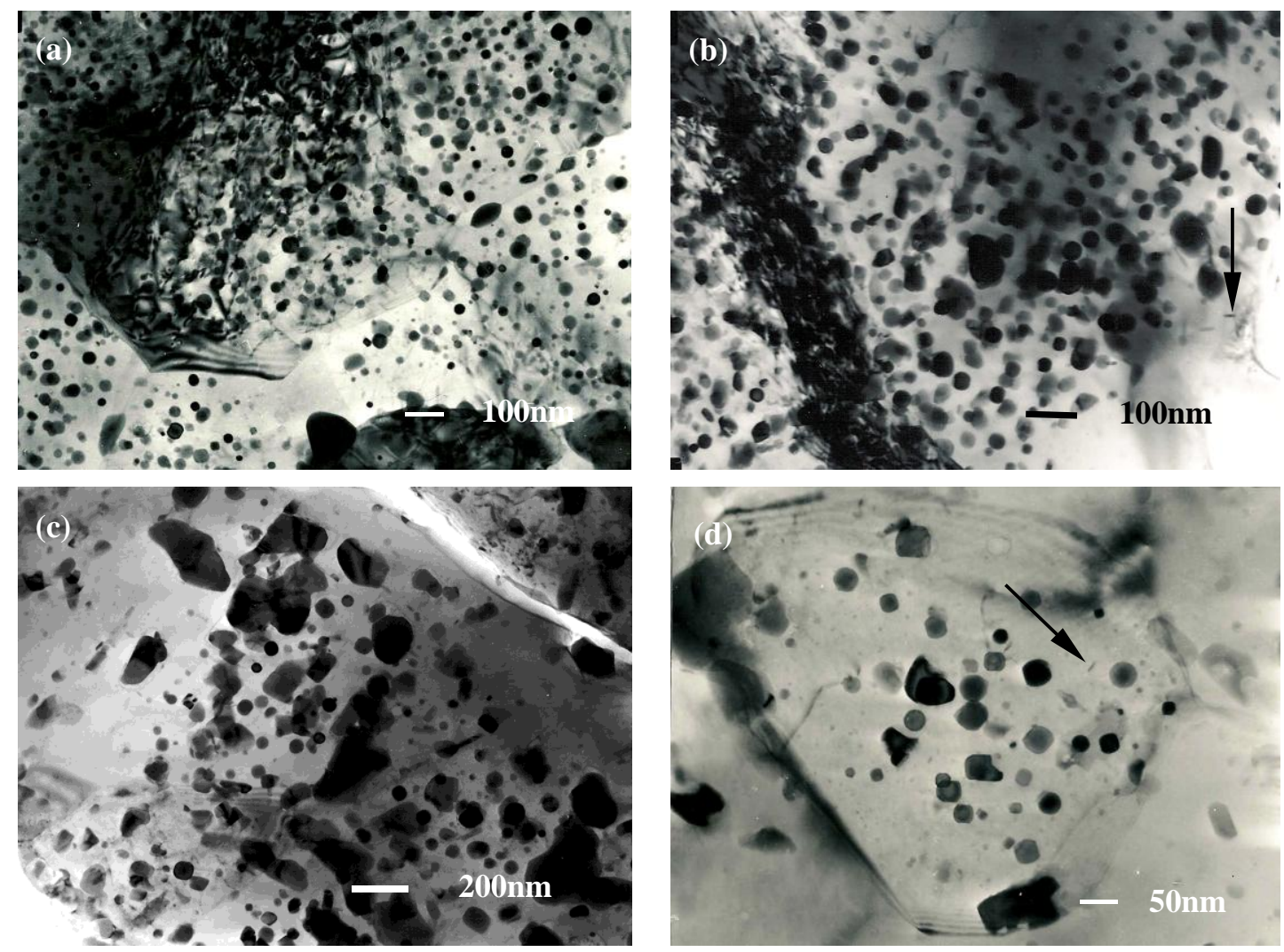

Figure 2 TEM images showing coarsening of nanoscale Sn particles with annealing temperature and increasing time. (a) As-sprayed coating. (b) The coating heat treated at $300^{\circ} \mathrm{C}$ for 1 hour. (c, d) Coating annealed at $300^{\circ} \mathrm{C}$ for 5 hours.

The TEM images of Figure 2 are used to illustrate the development of the nanoscale Sn particles with annealing and increasing annealing time. The nanoscale $\mathrm{Sn}$ particle dispersoid in the as-sprayed coating (Fig. 2a) coarsens slightly after 1 hour annealing at $300^{\circ} \mathrm{C}$ (Fig. 2b) while these particles further coarsen after 5 hours of annealing (Fig. $2 \mathrm{c}, \mathrm{d})$. It is noted that many nanoscale $\mathrm{Sn}$ particles are no longer spherical with annealing, but become elongated and irregular in shape within the Al matrix due to the merging of two or more particles. Larger Sn particles located at the grain boundaries similarly develop irregular shapes with annealing. In addition, annealing at $300^{\circ} \mathrm{C}$ induced the formation of very fine rod-like precipitates (e.g. Figs. 2 b,d, arrowed) which required EFTEM characterisation as follows.

The EEL spectrum from a rod-like precipitate and the matrix region of a sample annealed at $300^{\circ} \mathrm{C}$ for 1 hour shows that there are two $\mathrm{Cu}$ energy peaks $\left(\mathrm{L}_{3}\right.$ with energy $930 \mathrm{eV}$ and $\mathrm{L}_{2}$ with energy $951 \mathrm{eV}$ ) from the region of the precipitate (Fig. 3a). EFTEM maps for $\mathrm{Sn}, \mathrm{Cu}$ and $\mathrm{Al}$ were acquired from this feature at energies of $\mathrm{Sn}_{\mathrm{M}}(485 \mathrm{eV})$, $\mathrm{Cu}_{\mathrm{L}}(931 \mathrm{eV})$ and $\mathrm{Al}_{\mathrm{K}}(1560 \mathrm{eV})$, respectively (Figs. 3b-d). The maps delineated a precipitate of approximately $10 \mathrm{~nm}$ by $30 \mathrm{~nm}$ in size, rich in $\mathrm{Cu}$ and depleted in $\mathrm{Al}$, while no Sn was found to be present. Slight distortion of the Al map has occurred due to sample drift during the long exposure time. These results indicate that these fine rodlike precipitates are a Cu-Al compound. This is consistent with Porter et al (1981) who use phase diagrams to predict that $1 \mathrm{wt} . \% \mathrm{Cu}$ within the $\mathrm{Al}$ may form $\theta^{\prime}$ precipitates after annealing at $300^{\circ} \mathrm{C}$. No evidence of $\theta^{\prime}$ was found in the as-sprayed coatings, but it was identified in the coatings annealed at $300^{\circ} \mathrm{C}$ for 5 hour.

Returning to the phenomenon of Sn particle coarsening within the Al matrix and its relationship with the material hardness. It is noted that the melting points of pure $\mathrm{Sn}$ and 


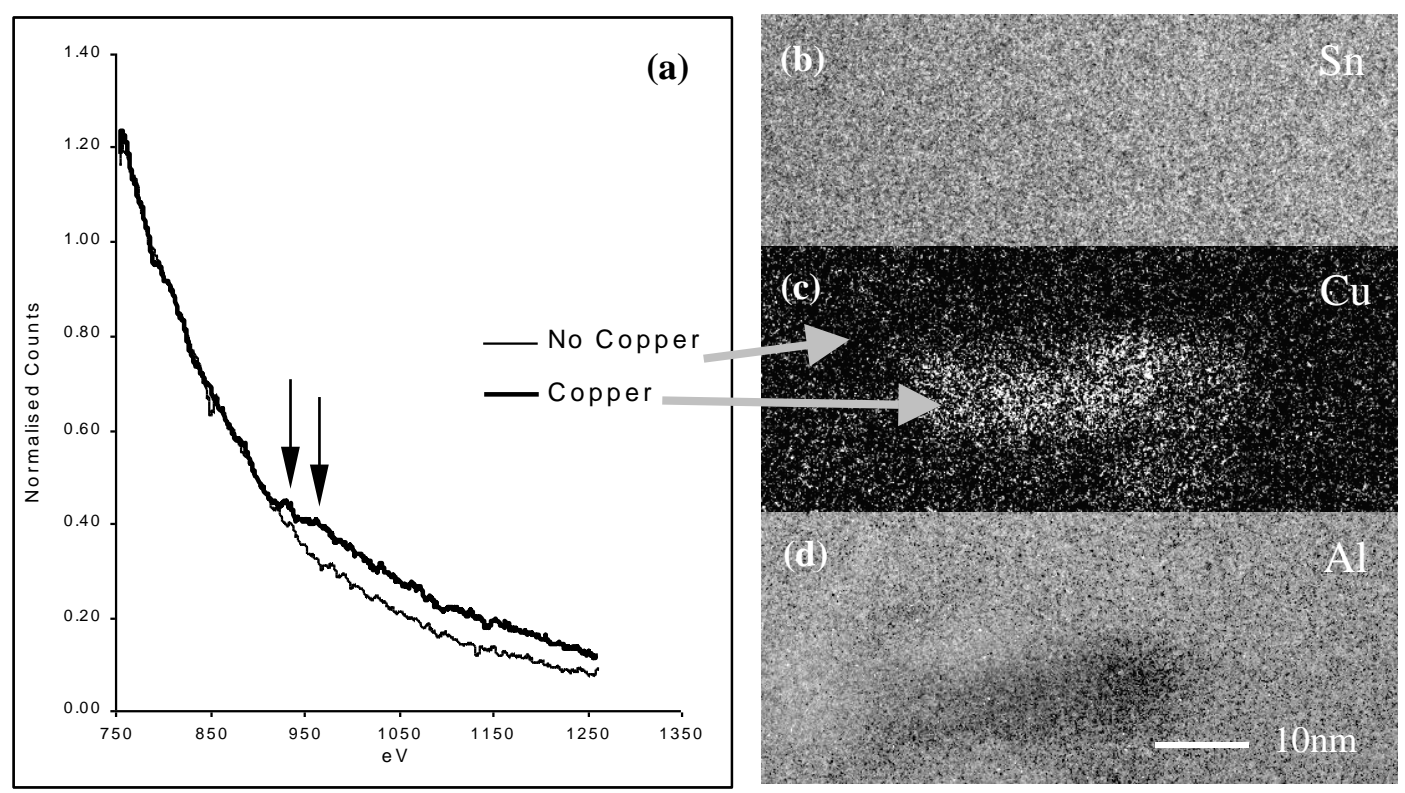

Figure 3 EELS spectrum and EFTEM result from an elongated precipitation. (a) EEL spectrum of matrix and precipitates; (b-d) EFTEM maps of $\mathrm{Sn}, \mathrm{Cu}$ and $\mathrm{Al}$ distribution.

$\mathrm{Al}$ are $232^{\circ} \mathrm{C}$ and $660^{\circ} \mathrm{C}$, respectively. It is thus expected that the sub-micron and nanoscale $\mathrm{Sn}$ particles adopt a liquid state in the solid $\mathrm{Al}$ matrix during annealing at $300^{\circ} \mathrm{C}$. The liquid elemental phase is predicted to move as a coherent droplet within the solid phase, driven by local temperature gradients (McLean et al, 1974). During the process of movement the liquid Sn droplet dissolves solid Al at the hot end of the particle and condenses $\mathrm{Al}$ at the trailing, cold end. When two or more liquid Sn droplets approach, they will start to merge to form a larger Sn particle. Surface energy of the coalescing Sn droplets will tend to make the irregular shapes more spherical given sufficient time at a high enough temperature. With increasing time of annealing, the nanoscale Sn particles tend to be eliminated, either by the coarsening of particles within the grains or by the coalescence of larger particles at the grain boundaries due to the additional effect of local strain fields.

The reason the microhardness decreases with increasing annealing time (Kong et al, 2001) is therefore attribute to this coarsening of the nanoscale and sub-micron Sn, combined with the local release of strain within the $\mathrm{Al}$ grains. The precipitation of $\theta^{\prime}$ might be expected to increase the microhardness of the annealed coatings by dispersion strengthening. However, the volume fraction of $\theta^{\prime}$ precipitates is not high enough to significantly effect the microhardness as compared with the effect of Sn.

\section{Conclusion}

The microhardness decrease with increasing time of annealing is attributed to the coarsening of sub-micron and nanoscale Sn particles. The precipitation of $\theta^{\prime}$ is additionally identified within the annealed coatings but this is not considered to significantly affect the microhardness. A liquid migration mechanism is invoked to explain the coarsening of $\mathrm{Sn}$ particles.

\section{References}

Kong CJ, Brown PD, Horlock AJ, Harris SJ and McCartney DG 2001 Inst. Phys. Conf. Ser., 227-230, 168

McLean M and Loveday MS 1974 Journal of Materials Science 1104-1114, 9

Porter DA and Eastering KE 1981 Phase Transformations in Metals and Alloys (Van Nostrand Reinhold (UK) Co. Ltd.) 291 